\title{
Ein transparentes Modell für einen fairen Preis für innovative Arzneimittel in Europa
}

Anne Hendrickx und Thomas Kanga-Tona

Inhaltsverzeichnis

11.1 Defizite der aktuellen Preisfestsetzungsverfahren für Arzneimittel - 157

11.2 Der Weg zu faireren Preisen - 158

11.3 Der Algorithmus der AIM für einen fairen Preis - 159

11.4 Besonderheiten und Stärken des AIM-Modells - 159

11.5 Parameter im Detail nebst Erläuterung - 160

11.5.1 F\&E - 160

11.5.2 Produktions- und Gemeinkosten - 163

11.5.3 Vertrieb und fachliche Information - 163

11.5.4 Grundgewinn - 164

11.5.5 Innovationsbonus - 164

11.5.6 Fairer Preis - 165

11.6 Anwendungsbereich und Limitationen des Modells - 165

11.7 Ergebnisse - 166

11.7.1 Weit verbreitete Krankheiten am Beispiel Hepatitis C - 166

11.7.2 Seltene Erkrankungen am Beispiel von SMA - 167 
11.7.3 Indikationsausweitung am Beispiel von NDM - 167

11.7.4 Kostentreiber Krebs - 168

11.8 Praktische Umsetzung - 168

11.8.1 Kurzfristig: Sensibilisierung und Anwendung des Systems auf lokaler Ebene - 168

11.8.2 Langfristig: Verknüpfung des fairen Preises mit der Marktzulassung auf EU-Ebene - 170

11.9 Der Weg nach vorn: Transparenz und Methodik - 171

11.10 Fazit -172

Literatur - 172 


\section{- Zusammenfassung}

Das empfindliche Gleichgewicht zwischen Bezahlbarkeit von Arzneimitteln, Nutzen für Gesundheitssysteme und Patient:innen sowie Einnahmen für die Pharmafirmen hat sich zugunsten letzterer eingependelt. Die europäischen Gesundheitssysteme tun sich schwer damit, Patient:innen zeitnahen Zugang zu neuartigen Arzneimitteln zu ermöglichen, weil deren hohe Preise nicht gerechtfertigt sind. Wie aber verschafft man den Patient:innen $\mathrm{Zu}$ gang und setzt dabei gleichzeitig Anreize für die Pharmaindustrie, in neue Arzneimittel zu investieren, die die Patient:innen auch wirklich brauchen? In ihrem Modell für eine faire Preisfestsetzung in Europa plädiert die Association Internationale de la Mutualité (AIM) für eine aktivere Rolle der Preisfestsetzungsund Erstattungsbehörden in Europa bei der Definition und Festsetzung ,fairer" Preise für innovative Arzneimittel. Alternativ zum bisherigen Value-based Pricing schlägt die AIM einen einfachen, aber effektiven Algorithmus vor, der auf die zugrunde liegenden Kosten und den therapeutischen Nutzen abstellt. Wenn man den Gewinn auf ein vernünftiges Maß beschränken und gleichzeitig relevante Innovationen honorieren würde, dann könnte man die Arzneimittelpreise senken und den Patient:innen einen besseren Zugang zu diesen Medikamenten verschaffen. Mit ihrem Modell für eine faire Preisfestsetzung in Europa leistet die AIM einen Beitrag zu einer zentralen Debatte, die die Gesundheitssysteme weltweit in den kommenden Jahren beschäftigen wird.

\subsection{Defizite der aktuellen Preisfestsetzungsverfahren für Arzneimittel}

Die heutigen Preisfestsetzungsverfahren für Arzneimittel folgen einer Reihe unzureichender Prinzipien und fehlerhafter Mechanismen. Tatsächlich tragen Value-based Pricing und die Intransparenz bei den inzwischen weitverbreiteten sogenannten Managed Entry Agreements dazu bei, dass die Preisfestsetzung für innovative Produkte eine schwierige Angelegenheit ist (Wenzl und Chapman 2019). Preisfestsetzungsverfahren sind komplex und mit ausgeklügelten Health Technology Assessments (HTA) und mühsamen Diskussionen über die „Zahlungsbereitschaft“" verbunden. Die Regierungen bemühen sich eigentlich, Arzneimittelpreise festzusetzen, die den Bedürfnissen der Gesundheitssysteme gerecht werden, die Wirksamkeit der Arzneimittel widerspiegeln und gleichzeitig das Budget im Zaum halten. Doch der Mangel an Informationen über die Wirksamkeit des Produkts, fehlende Informationen über die Forschungs- und Entwicklungskosten der Unternehmen sowie nicht offengelegte Nettopreise erschweren dies. Zahlreiche Arzneimittel überschreiten die Kosteneffektivitätsschwellen vieler Ländern bei Weitem, sodass dort aus den Schwellen tendenziell wohl eher Untergrenzen geworden sind.

Als Value-based Pricing eingeführt wurde, schien es eine gute Methode, um die finanziellen Mittel der Gesundheitssysteme für die Therapien mit dem höchsten Nutzen einzusetzen. In der Praxis war seine Anwendung allerdings mit einer Reihe von nachteiligen Folgen für die Gesundheitssysteme und den Zugang der Patient:innen zu Arzneimitteln verbunden. Erstens hat es den langfristigen Preisanstieg bei Arzneimitteln nicht verhindert. Das gilt insbesondere für Spezialgebiete wie die Onkologie (Morgan et al. 2020). Zweitens hat Value-based Pricing die Kosten der Hersteller für die Entwicklung ihrer Medikamente völlig von den Preisen entkoppelt, die die Gesundheitssysteme dafür zahlen; das wiederum hat „profitorientiertes“ Verhalten ermöglicht. Drittens führen die extrem hohen Preise, die dieses System den Unternehmen potenziell gewährt, dazu, dass der Zugang zu Arzneimitteln in manchen Gesundheitssystemen inzwischen rationiert werden muss.

Zudem sollte man im Preisfestsetzungsverfahren für Arzneimittel nicht die kognitive Verzerrung bei der Entscheidungsfindung unterschätzen. Diese besteht darin, zu sehr auf die erste angebotene Information (den „An- 
ker“) zu vertrauen - der sogenannte „AnkerEffekt“ (Harvard Law School 2019). Oft gibt der von der Pharmafirma aufgerufene Preis den Rahmen vor, innerhalb dessen diskutiert wird. Das gilt insbesondere für Orphan Drugs, die ja keiner Kosten-Nutzen-Analyse unterworfen sind. Diese Preisfestsetzungsmethode lässt sich sogar durch frühzeitige und völlig unrealistisch hohe Preisankündigungen der Unternehmen für künftige Therapien (Bach 2019) verstärken, die in den Köpfen der Käufer hohe Kostenerwartungen wecken und damit jeden angemessenen Preis zu einem späteren Zeitpunkt verhindern.

Sich allein auf den Nutzen zu verlassen hat $\mathrm{zu}$ inflationären Preisen geführt und die Preisfestsetzungs- und Erstattungsinstitutionen so weit in die Ecke gedrängt, dass sie jeden Preis akzeptieren. Darüber hinaus erlauben die beträchtlichen Umsätze, die große Unternehmen durch hohe Preise erzielen, sehr hochpreisige Übernahmen kleinerer Unternehmen, die vielversprechende Arzneimittel entwickeln. Das wiederum führt zu neuen Preissteigerungen. Um diesem inflationären Trend Einhalt zu gebieten, gilt es einen objektiveren Ausgangspunkt für Preisverhandlungen festzulegen. Dieser muss den Nutzen des Produkts einbeziehen, sollte ihn aber auch nicht zur alleinigen Grundlage machen.

Es ist daher nötig, weitere Kriterien einzuführen, um die Unklarheiten bei der Preisfestsetzung möglichst $\mathrm{zu}$ beseitigen und $\mathrm{zu}$ den Grundlagen des Patentsystems zurückzukehren: Kosten für Forschung und Entwicklung wieder einzuspielen und eine angemessene Rendite zu erwirtschaften.

\subsection{Der Weg zu faireren Preisen}

Das Konzept der fairen Arzneimittelpreise ist in den vergangenen Jahren immer wichtiger geworden und hat sowohl in Europa als auch im Rest der Welt in politischen Debatten an Bedeutung gewonnen. Befürworter dieses Ansatzes erkennen an, dass Arznei- mittel ein Eckpfeiler der Gesundheitssysteme sind und dass Gesundheitssysteme natürlich auch die Pharmahersteller als feste Lieferanten innovativer Arzneimittel brauchen. Allerdings versuchen die Unterstützer fairer Preise das Risiko zu senken, dass Pharmaunternehmen ihr Marktmonopol ausnutzen (Akcigit et al. 2021), indem sie Preise festlegen, die lediglich widerspiegeln, was aus dem Markt herauszuholen ist. Die Befürworter des Ansatzes beachten auch, dass hohe Arzneimittelpreise aufgrund von Rationierung und späterer Verfügbarkeit dazu führen, dass $\mathrm{Pa}$ tient:innen und Gesundheitssysteme nicht den vollen gesellschaftlichen Nutzen aus pharmazeutischen Innovationen ziehen können. $\mathrm{Zu}-$ dem kann Arzneimittelpreisregulierung auch dazu dienen, unangemessen hohe Einnahmen für Produkte mit geringem Zusatznutzen zu reduzieren. Die erwartbaren Vorzüge fairer Arzneimittelpreise sind aus der Perspektive des Gesundheitswesens klar und viele Organisationen befassen sich bereits mit diesem Konzept. Die Weltgesundheitsorganisation (WHO) definierte den fairen Preis im Jahr 2017 als ,...) einen Preis, der erschwinglich für die Gesundheitssysteme und Patient:innen ist und gleichzeitig genug Marktanreize setzt, damit die Industrie in Innovationen und Arzneimittelproduktion investiert." (WHO 2017)

Ein fairer Preis muss, konkret gesagt, ein Gleichgewicht schaffen zwischen der Notwendigkeit,

- Preise auf einem Niveau festzusetzen, das den Zugang nicht verhindert. Das bedeutet wiederum, eine Verbindung zwischen dem Preis einerseits und den Kosten für die Entwicklung und Produktion von Arzneimitteln andererseits wiederherzustellen;

- eine vernünftige Investitionsrendite zu ermöglichen, sodass die Pharmafirmen weiter Innovationen herstellen. Dabei wird gleichzeitig ein klares Signal gesendet, was ein akzeptables Ausgabenniveau ist, insbesondere in Bezug auf Firmenübernahmen; 
- Forschung und Entwicklung zu motivieren, Innovationen herzustellen, die die Gesundheitssysteme brauchen und die einen therapeutischen Nutzen für die Patient:innen haben, insbesondere dort, wo noch ungedeckter Bedarf besteht.

\subsection{Der Algorithmus der AIM für einen fairen Preis}

Die Association Internationale de la Mutualité (AIM) hat ein faires Preisfestsetzungsmodell entwickelt, das auf einem einfachen, aber effektiven Algorithmus basiert. Dieser ermöglicht es, einen europäischen fairen Preis für jedwedes innovative Arzneimittel zu kalkulieren. ${ }^{1}$

Im Modell wird grundsätzlich jede neue Substanz oder neue Indikation, die künftig mithilfe eines bereits bestehenden Wirkstoffs behandelt werden soll, als innovatives Arzneimittel gewertet. Dabei ist es unerheblich, ob Wirksamkeit und Zweckmäßigkeit nachgewiesen sind oder nicht.

Nutzt man den Algorithmus, ergibt sich der faire Preis eines Arzneimittels pro Patient:in und Behandlung aus der Summe folgender Komponenten: (• Abb. 11.1):

- Kosten für das Pharmaunternehmen: Forschung und Entwicklung (F\&E) pro Patient:in, Produktionskosten pro Behandlung, Anteil an Gemeinkosten und Vertrieb und medizinischer Information
- Ein angemessener Grundgewinn (basic profit) für den Zulassungsinhaber in Bezug auf diese Kosten

- An den therapeutischen Nutzen gekoppelter ,Innovationsbonus“, der sich auf bis zu $40 \%$ zusätzlichen Gewinn summieren kann

\subsection{Besonderheiten und Stärken des AIM-Modells}

Viele Akteure im Gesundheitswesen wie die WHO, die europäische Organisation für seltene Erkrankungen Eurordis und die European Cancer Leagues haben über Alternativmodelle zum aktuellen Value-based Pricing nachgedacht. Die Ergebnisse und Lösungselemente weisen viele Ähnlichkeiten mit dem AIM-Modell auf. Allerdings ist das AIM-Modell in mehrerlei Hinsicht einzigartig.

\section{- - Setzt keine Transparenz voraus}

Auch wenn die Bemühungen um Kostentransparenz (insbesondere bei F \& E) dem Modell und den Gesundheitssystemen insgesamt zugutekommen würden, setzt es diese nicht voraus. Es plant vielmehr schon einen möglichen Mangel an Bereitschaft der Unternehmen zur Offenlegung sowie die Komplexität bei der Identifizierung von Kosten ein. Mehr noch: Der faire Preis kann sogar ganz ohne Zusammenarbeit mit dem pharmazeutischen Sektor ermittelt werden. Sind echte Zahlen schwer zu

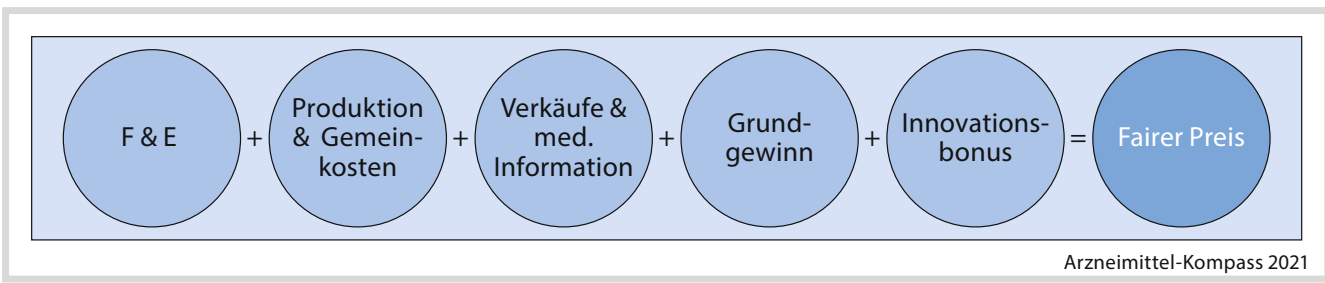

- Abb. 11.1 Der AIM-Algorithmus für faire Preise

$1>$ https://www.aim-mutual.org/mediaroom/aimpresents-its-model-for-fair-and-transparent-pricesfor-pharmaceuticals/. 
ermitteln, werden Pauschalbeträge oder feste Prozentzahlen genutzt.

\section{- - Der Innovationsbonus - ein flexibles Werkzeug, um sinnvolle Innovation zu fördern}

Um Forschungsaktivitäten an Innovationen zu fördern, die den Patient:innen auch wirklich etwas bringen, beinhaltet das Modell den sogenannten Innovationsbonus: einen zusätzlichen Gewinn für den Pharmahersteller, wenn er ein Arzneimittel entsprechend seiner therapeutischen Leistungsfähigkeit und dem gesellschaftlichen Bedarf auf den Markt bringt. Derzeit lautet der Vorschlag, den Bonus mit drei Dingen zu verknüpfen: der Schwere der Krankheit, dem (ungedeckten) medizinischen Bedarf und dem therapeutischen Nutzen (ausgedrückt in festen Endpunkten). Der Bonus könnte auch mit anderen Aspekten verbunden werden, die für nachhaltige innovative Arzneimittel gelten: mit der Datenqualität (also doppelblinden randomisierten klinischen Versuchen), einer spezifischen Zielpopulation (Kinder, ältere Menschen etc.) oder anderen relevanten Zielen wie etwa dem Produktionsstandort.

\section{- - Ein „konkreter" Preis}

Bislang ist das AIM-Modell das einzige, das einen konkret kalkulierten fairen Preis vorschlägt, der einen sofortigen Vergleich mit dem aktuellen (Listen-)Preis oder dem vom Pharmaunternehmen vorgeschlagenen Preis ermöglicht.

\subsection{Parameter im Detail nebst Erläuterung}

Um die derzeitige Nichtverfügbarkeit von Daten zu Unternehmenskosten, beispielsweise für F \& E, zu kompensieren, verwendet das Modell feste Parameter wie Pauschalbeträge oder Prozentsätze. Außerdem setzt es auf Parameter mit sehr begrenzten Auswahlmöglichkeiten. Dies verschafft Nichtfachleu- ten eine Orientierungshilfe und bietet Fachleuten, die sich auf das Modell stützen wollen, einen guten Ausgangspunkt für Diskussionen über die Akzeptanz der vorgeschlagenen Parameter.

Im Falle einer künftigen Einführung des Modells müssen die Parameter jedoch noch weiter verfeinert und diskutiert werden, und zwar sowohl auf der technischen als auch der politischen Ebene.

Die im Modell getroffenen Entscheidungen und die dahinterstehenden Überlegungen werden im Folgenden beschrieben.

\subsubsection{F \& E}

a) $\mathbf{F} \& \mathbf{E}$ bei $\mathbf{E U}-27-S t a a t e n=$ Pauschalbetrag von 250 Millionen $€$ oder tatsächliche F \& E weltweit (dokumentiert) - max. 2,5 Mrd. $€$

$\times$ Anteil der EU-27-Staaten: 35,85\%

Das Ziel des Modells ist es, Fairness gegenüber den Kostenträgern und den Pharmaunternehmen herzustellen. Geld, das in Forschung gesteckt wurde, sollte wieder zurückerstattet werden. Aber das Modell sollte auch Anreize für Effizienz bei den Investitionen setzen und die Inhouse-Entwicklung neuer Wirkstoffe und sinnvolle Investitionen statt überteuerter spekulativer Übernahmen belohnen. Deswegen sollte es einen Grenzwert für die akzeptierte Summe geben sowie eine klare Definition, was denn überhaupt unter $\mathrm{F} \& \mathrm{E}$ fällt.

Der im Modell vorgesehene Gesamtbetrag für $F \&$ E reicht von 250 Mio. $€$ bis 2,5 Mrd. $€$ und beinhaltet

- eine Pauschale von 250 Mio. $€$ : Laut einigen Quellen (van der Gronde et al. 2017) deckt diese Summe die untere Spanne von Forschungsinvestitionen ab, die erforderlich sind, um ein neues Arzneimittel auf 
den Markt zu bringen. Diese Pauschale sollte daher ausreichen, wenn F\&E des Unternehmens oder der Organisation effizient sind. Sie könnte sogar mehr abdecken als die realen Kosten und damit den effizienten Umgang mit Finanzmitteln stimulieren und belohnen.

- reale Kosten: Den Unternehmen können dokumentieren, dass sie mehr in $\mathbf{F} \& \mathbf{E}$ investiert haben und nun den realen $\mathrm{Be}$ trag zurückzuverlangen, den sie weltweit in F \& E hinsichtlich des spezifischen Arzneimittels oder der Indikation gesteckt haben. Der Gesamtbetrag, den sie verlangen können, wird jedoch auf 2,5 Mrd. $€$ begrenzt. Diese Summe gilt heute als oberer Kostenbereich (Prasad und Mailankody 2017). Diese im Vergleich zu den extrem hohen Übernahmekosten - bisweilen um oder über zehn Milliarden US-Dollar 2,3,4,5,6 vergleichsweise moderate Summe soll angemessenere Firmenübernahmen und mehr Inhouse-Produktentwicklung befördern.

2 Gilead übernahm Kite pharma für 11,9 Mrd. USDollar; https://www.gilead.com/news-and-press/ press-room/press-releases/2017/8/gilead-sciencesto-acquire-kite-pharma-for-119-billion.

3 Celgene übernahm Juno Therapeutics für $9 \mathrm{Mrd}$. US-Dollar; https://www.celgene.com/newsroom/ cellular-immunotherapies/celgene-corporation-toacquire-juno-therapeutics-inc/.

4 GSK übernahm Tesaro für 5,1 Mrd. US-Dollar; > https://www.gsk.com/en-gb/media/press-releases/ gsk-completes-acquisition-of-tesaro-an-oncologyfocused-biopharmaceutical-company/\#.

5 Eli Lilly übernahm Loxo Oncology für $8 \mathrm{Mrd}$. USDollar; https://investor.lilly.com/news-releases/ news-release-details/lilly-announces-agreementacquire-loxo-oncology.

6 Novartis übernahm AveXis für 8,7 Mrd. US-Dollar; - https://www.novartis.com/news/media-releases/ novartis-enters-agreement-acquire-avexis-inc-usd87-bn-transform-care-sma-and-expand-positiongene-therapy-and-neuroscience-leader; $>$ https:// www.novartis.com/news/media-releases/novartissuccessfully-completes-acquisition-avexis-inc.
Die F \& E-Kosten im Modell beinhalten jedwede Ausgaben bis hin zur Marktzulassung (auf europäischer Ebene); direkte Kosten oder „Out of Pocket“-Eigenbeteiligungen sowie indirekte F \& E-Kosten (Lizenzgebühren, Übernahmen etc.) für präklinische und klinische Entwicklung und alle Ausgaben in Zusammenhang mit $\mathrm{F} \& \mathrm{E}$ bis hin zur Zulassung. Es gibt keine allgemein konsentierte Methode zur Berechnung derartiger Kosten. Eine Methodik für ein transparentes Berichtswesen für Forschungskosten, das Doppelverbuchungen vermeidet, muss erst noch entwickelt werden. Vorzugsweise sollte es auf akademischer Forschung basieren. Es müssen komplexe Entscheidungen zu sensiblen Fragestellungen gefällt werden. Das schließt auch ein, wie Ausgaben berücksichtigt werden können, die eigentlich nicht von den Sponsoren übernommen werden, zum Beispiel öffentlich finanzierte Forschung, Steuervorteile und Opportunitätskosten. Ebenso fällt der Anteil der Unternehmensinvestitionen durch Übernahme darunter, der F \& E zugerechnet werden kann. Um den Risikofaktor bei der Arzneimittelentwicklung einzubeziehen, müssen die Ausfallkosten für Arzneimittel, die es nicht bis zur Marktreife geschafft haben, in angemessener Weise durch diejenigen ausgeglichen werden, die es geschafft haben.

Die Bevölkerung der EU27-Staaten entspricht 35,85\% der Bevölkerung der entwickelten Märkte für innovative Arzneimittel (AIM 2021). Im Modell wird dies als Anteil Europas an der weltweiten F \& E betrachtet. Obwohl Länder, die allmählich einen Pharmasektor ausbilden, Zugang zu diesen neuen Therapien haben, sollte dies nicht den Anteil beeinflussen, den Europa aus internationaler Solidarität im weiteren Sinne zahlt. 
b) Zahl der Patient:innen mit der Indikation (zehn Jahre) = Seltene oder Prävalenz in EU-27-Staaten chronische (je 10.000 oder Bevölkerung) Krankheit: Krebs: Inzidenz in EU-27-Staaten $\times$ zehn Jahre Patentschutz $\times$ Behand- $50 \%$ lungsrate $\times$ Marktanteil (max. zwei Wettbewerber)

Die kalkulierten EU27-F \& E-Kosten sollten innerhalb der Patentlaufzeit wieder eingespielt werden, inklusive Primärpatent und weiterer IP-Schutzmaßnahmen für das spezifische Arzneimittel oder die spezifische Indikation. Im Modell wird die verbleibende Patentschutzdauer mit zehn Jahren angesetzt und liegt damit unter der durchschnittlichen Patentschutzdauer von 12-13 Jahren (EFPIA 2019) nach dem Marktzugang.

Die Anzahl der Patient:innen, die in diesen zehn Jahren behandelt werden, wird für die einzelnen Indikationen berechnet. Dies können alle sein, die an der entsprechenden Krankheit leiden, oder der Anteil, der an einem Untertyp der Krankheit leidet, ein Behandlungsstrang oder eine bestimmte Altersgruppe.

Im ersten Schritt wird die theoretische Zielgruppe mittels Prävalenz oder Jahresinzidenz der Indikation ${ }^{7}$ bestimmt. Vorzugsweise werden dabei - soweit verfügbar - europäische Daten genutzt. Prävalenz bzw. Inzidenz werden dabei je nach Art der Erkrankung erheblich variieren; von weniger als 2/100.000 für

7 Bei (äußerst) seltenen chronischen Krankheiten wird gewöhnlich die Prävalenz herangezogen, also die Anzahl der Patient:innen zu einem bestimmten Zeitpunkt. Die Frage lautet: „Wie viele Menschen leiden gerade im Moment an dieser Krankheit?" Bei Krebs nutzt man hingegen die Inzidenz, also die Anzahl der Menschen, die diese Krankheit in einem bestimmten Jahr betrifft. Die Frage lautet hier: „Wie viele Menschen werden in diesem Jahr von dieser Krankheit betroffen sein?". eine äußerst seltene Krankheit bis hin zu mehr als $5 \%$ für sehr häufige Krankheiten.

Im zweiten Schritt wird auf diese theoretische Zielgruppe eine „Behandlungsrate“ angewandt. Die nationalen Gegebenheiten wie beispielsweise Zugangsbedingungen, Fehldiagnosen, mangelnde Infrastruktur in einigen Mitgliedsstaaten oder das Versterben von Patient:innen vor dem Zugang zur Behandlung werden im Modell geschätzt. So wird einberechnet, dass nur durchschnittlich $50 \%$ der Zielpopulation der EU27-Staaten behandelt werden.

Zudem - und das ist häufig der Fall - ist das neue Medikament unter Umständen nicht das einzige, das auf die Indikation abzielt. Es muss mithilfe eines Horizon Scanning sondiert werden, ob und wenn ja welche kurz- und mittelfristigen konkurrierenden Marktzugänge zu erwarten sind. Das Modell ermöglicht die Berücksichtigung von bis zu zwei weiteren Mitbewerbern pro neues Arzneimittel. Selbst wenn es in einigen Fällen mehr sind, geht das Modell von der Annahme aus, dass das erste Pharmaunternehmen, dessen Produkt auf dem Markt erscheint, nicht weniger als ein Drittel des europäischen Marktes abdeckt. Der Markt wird zu gleichen Teilen unter den Mitbewerbern aufgeteilt. Im Falle von Märkten mit Mono- oder Kombinationstherapien, etwa gegen antivirale Hepatitis $\mathrm{C}$ oder in vielen $\mathrm{Be}-$ reichen der Onkologie, sollten der Mitbewerber und der dazugehörige Marktanteil auf der Unternehmensebene betrachtet werden, nicht auf der Ebene der einzelnen Produkte.

1. $\quad \mathbf{F} \& \mathbf{E} /$ Patient:in (pro Behandlung) = F \& E EU-27 (a)/Patient:innen mit der Indikation (b)

Die jedem europäischen Patienten zugeordneten Kosten entsprechen dem F \& E-Anteil in Europa geteilt durch die Anzahl der Patient:innen in der EU27. 


\subsubsection{Produktions- und Gemeinkosten}

\section{Produktions- und Gemeinkosten $=$}

Kosten/Monat: reale Kosten

(dokumentiert) oder Pauschale:

Normale Arznei- $\quad 50 €$ für chemische

mittel

oder

$150 €$ für biologische

Orphan Drug $\quad 250 €$ für chemische

oder

$750 €$ für biologische

$\times$ Dauer der Behandlung in Monaten

Das zweite Element des fairen Preises sind die Produktions- und Gemeinkosten. Diese schließen die Herstellungskosten ein: Warenkosten, Produktion inklusive Investitionen in Produktionsstätten und Verpackung ebenso wie Vertriebskosten inklusive Lagerhaltung und Transport, Steuern, direkte und indirekte Kosten wie Buchhaltung, Bestellwesen oder Versicherung.

Unter Anwendung derselben Methodik wie bei den F \& E-Kosten wird eine Pauschale von $50 €$ pro Behandlungsmonat angesetzt, es sei denn, der Inhaber der Marktzulassung weist höhere tatsächliche Kosten nach. Um die Kosten abzubilden, die mit der Komplexität der biologischen Arzneimittelproduktion verbunden sind, setzt das Modell eine Pauschale von $150 €$ für diese besonderen Arzneimittel an. Für spezielle Arzneimittel wie Impfstoffe sollten die realen Kosten angesetzt werden. Außerdem geht das Modell davon aus, dass die Kosten der Herstellung von Orphan Drugs fünfmal höher liegen, um deren geringes Umsatzvolumen zu kompensieren. Aufgrund von Skaleneffekten können die Kosten für Krankheiten mit hoher Prävalenz oder Arzneimittel mit geänderter Indikation, die oft weit verbreitet und seit langem auf dem Markt sind, abgesenkt werden. Die Produktion in der EU ist möglicherweise auch ein Faktor, der die Kosten erhöht und mit einer spezifischen Pauschale berücksichtigt werden könnte.

Die monatlichen Kosten werden multipliziert mit der durchschnittlichen Dauer der Behandlungsschwerpunkte, ausgedrückt in Behandlungsmonaten. Bei der Behandlung chronischer Krankheiten berücksichtigt das Modell eine Dauer von zehn Jahren für alle Therapien, was der Restlaufzeit des Patents entspricht.

Bei Gen- oder Zelltherapien setzt das Modell $60.000 €$ an; dies entspricht der Obergrenze der Produktionskosten von CAR-T-Zellen in einer akademischen Non-Profit-Umgebung in Deutschland (Ran et al. 2020).

\subsubsection{Vertrieb und fachliche Information}

\section{Vertrieb und fachliche Information = \\ $20 \%$ der F \& E-Kosten}

Abschließend sind in Zusammenhang mit der Markteinführung eines neuen Medikaments weitere Kosten zu berücksichtigten. Dies schließt alle Kosten ein, die mit der Vermarktung des Arzneimittels vor Ort zu tun haben, inklusive Zulassung in unterschiedlichen Ländern, Verwaltung und Überwachung nach der Zulassung in Hinblick auf unerwünschte Wirkungen (beispielsweise Pharmakovigilanzsysteme, Phase-IV-Studien), ebenso wie fachliche Informationen für die verordnenden Ärztinnen und Ärzte und andere Akteure im Gesundheitswesen. Marketingaktivitäten werden im Modell nicht berücksichtigt; das Modell verknüpft diese Kosten mit F \& E und geht davon aus, dass ein Produkt, das teurer in der Entwicklung ist, auch Mehrkosten bei der Marktzugangsanalyse und der fachlichen Information verursachen könnte. Daher wird diesen Aktivitäten ein fixer Prozentsatz von $20 \%$ der F \& E-Kosten zugeordnet. 


\subsubsection{Grundgewinn}

4. Grundgewinn =

$8 \%$ der Kosten (F \& E + Produktion bzw.

Gemeinkosten + Verkäufe bzw. fachliche

Information)

Unternehmen müssen eine ausreichende Kapitalrendite erwirtschaften, um ihr Geschäft aufrechtzuerhalten. Das Modell berücksichtigt einen Grundbruttobetrag (basic gross profit) von $8 \%$ der Gesamtkosten als angemessenes Grundgewinnniveau (basic profit level), was im oberen Renditebereich risikobehafteter Wirtschaftszweige liegt und daher angemessen erscheint (Popa et al. 2018).

\subsubsection{Innovationsbonus}

Um Anreize zu setzen, dass Produkte eingeführt werden, die die Patient:innen auch wirklich brauchen, beinhaltet das Modell einen Innovationsbonus, der bei der Preisfestsetzung für Arzneimittel für den thera- peutischen Zusatznutzen steht. Der Hersteller kann ein Health Technology Assessment (HTA) vorschlagen, das den erwarteten therapeutischen Nutzen des Arzneimittels für diese Indikation belegt. Ein Innovationsbonus, der mit der Krankheitsschwere, dem (noch ungedeckten) medizinischen Bedarf und harten klinischen Endpunkten verknüpft ist, wird zum fairen Preis addiert. Ist tatsächlich ein Innovationsbonus zu veranschlagen, beträgt dieser zwischen 5 und $40 \%$ der Kosten.

Der Bonus wird anhand der folgenden Kriterien kalkuliert (• Abb. 11.2).

Wie bei den übrigen Parametern ist auch hier die verwendete Methodik wichtig. Es sollten bereits existierende Maßstäbe wie der European Society for Medical Oncology's Magnitude of Clinical Benefit Scale (ESMOMCBS) für Onkologika verwendet werden. Die Wahl des Vergleichsmedikaments (Placebo oder direkter Vergleichskandidat) ist hierbei entscheidend. Da der Innovationsbonus für jede neue Substanz kalkuliert wird, erhalten Mitbewerber gewöhnlich keinen Bonus in gleicher Höhe. Dies dient dem Ziel des Modells, die Zahl der Doppelentwicklungen zu senken und die Forschung in neuen Krankheitsbereichen anzuregen.

In den Innovationsbonus können weitere Kriterien einfließen, beispielsweise die Datenqualität, d.h. doppelblinde randomisierte klinische Studien, bestimmte sensible Zielpopulationen (Kinder, ältere Menschen ...), der Produktionsstandort etc. Der Innovationsbonus ist ein flexibles, sich entwickelndes Instrument. 


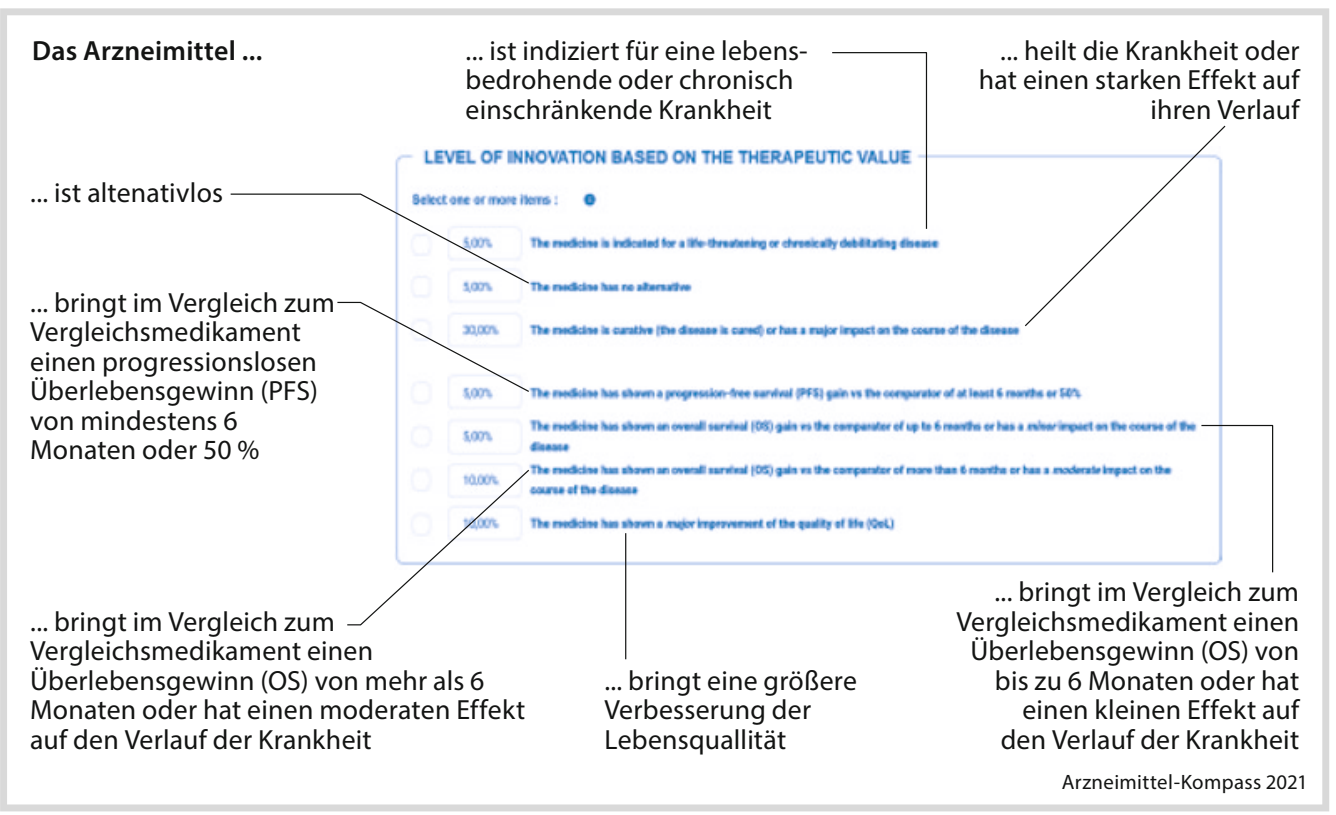

- Abb. 11.2 Innovationsbonus im Kalkulator auf der AIM-Website

\subsubsection{Fairer Preis}

Fairer Preis für EU 27-Staaten pro Behandlung pro Patient:in

$$
=1+2+3+4+5
$$

Der Algorithmus ermittelt einen Preis pro behandelte Patientin bzw. behandelten Patienten. Dies gewährt Unternehmen und Kostenträgern Vorhersagbarkeit: So wissen beide, was das Arzneimittel kosten wird.

Der faire Preis lässt sich einfach als Preis pro Behandlungsmonat oder Behandlungsjahr angeben.

Das Modell kalkuliert einen einheitlichen Preis für Europa, genauer: die EU 27Staaten, aber es ist auch möglich, einen Mechanismus zu entwickeln, um ihn an die Kaufkraft im jeweiligen Land anzupassen (AIM 2021).

\subsection{Anwendungsbereich und Limitationen des Modells}

Das Modell wurde für innovative Arzneimittel mit einem hohen Preis und nicht für Arzneimittel mit mehreren Anbietern entwickelt.

Seine Anwendung auf Erstanwärter für eine neue Indikation ist einfach und ermöglicht es, einen angemessenen Preis für die gesamte therapeutische Klasse festzusetzen. Nichtsdestotrotz bietet das Modell auch eine klare Methodik für komplexere Situationen wie Zweitindikationen, Zweitanwärter, Indikationsänderungen oder Kombinationstherapien, wobei bestimmte Kriterien berücksichtigt werden (AIM 2021).

Jedoch sind relevante Mechanismen auf EU-Ebene, die bislang noch nicht existieren, eine Voraussetzung dafür, um diesen radikal anderen Preisfestsetzungsansatz durchzusetzen. Würde man das Modell nur in einer kleineren Gruppe von Ländern einsetzen, wäre es weniger effektiv, denn dann könnten die 
Unternehmen entscheiden, die entsprechenden Märkte nicht zu beliefern.

\subsection{Ergebnisse}

Der mit dem Modell und Algorithmus von AIM kalkulierte faire Preis beträgt zumeist nur ein Zehntel des aktuellen Preises, wie die folgenden Beispiele zeigen, die aufgrund gut dokumentierter Kosteninformationen zusammengestellt wurden (vgl. • Tab. 11.1).

\subsubsection{Weit verbreitete Krankheiten am Beispiel Hepatitis C}

Die jüngste Generation antiviraler HepatitisC-Therapien ist ein gutes Beispiel dafür, wie das Modell bei einem leicht zu produzierenden Arzneimittel für eine weitverbreitete Krankheit in einem Bereich mit hoher Wettbewerbsintensität (drei pharmazeutische Unternehmen teilen sich den Markt für Mono- und Kombinationstherapien) funktioniert.

- Tab. 11.1 Beispiele für die Verwendung des AIM-Algorithmus

\begin{tabular}{|c|c|c|c|c|}
\hline Erkrankung/Indikation & Hepatitis C & $\begin{array}{l}\text { 5q Spinale mus- } \\
\text { kuläre Atrophie }\end{array}$ & $\begin{array}{l}\text { Nondystrophische } \\
\text { Myotonie }\end{array}$ & Prostatakrebs \\
\hline Prävalenz EU27 & $\begin{array}{l}1 \% \text { der Bevöl- } \\
\text { kerung }\end{array}$ & $2 / 100.000$ & $2,6 / 100.000$ & $140.000 / \mathrm{Jahr}$ \\
\hline Wettbewerber & 2 & 1 & - & 2 \\
\hline $\begin{array}{l}\text { Mit dem Arzneimittel behandelte } \\
\text { Patient:innen/10 Jahre }\end{array}$ & 745.833 & 2.238 & 5.818 & 233.333 \\
\hline Weltweite F \& E & $800.000 .000 €$ & $1.000 .000 .000 €$ & $292.887 .029 €$ & $473.300 .000 €$ \\
\hline 1. F \& E/Patient:in & $384,54 €$ & $160.223,46 €$ & $18.048,99 €$ & $727,19 €$ \\
\hline Produktion/Monat & $50 €$ & $250 €$ & $250 €$ & $250 €$ \\
\hline Behandlungsmonate & 3 & 120 & 120 & 8 \\
\hline 2. Produktion/Behandlung & $150 €$ & $30.000 €$ & $30.000 €$ & $2.000 €$ \\
\hline 3. Vertrieb/fachliche Information & $76,91 €$ & $32.044,69 €$ & $3.609,80 €$ & $145,44 €$ \\
\hline Gesamtkosten & $611,44 €$ & $222.268,16 €$ & $51.658,79 €$ & $2.872,63 €$ \\
\hline 4. Grundgewinn (8\%) & $48,92 €$ & $17.781,45 €$ & $4.132,70 €$ & $229,81 €$ \\
\hline Innovationsbonus & $40 \%$ & $15 \%$ & $20 \%$ & $20 \%$ \\
\hline 5. Innovationsbonus & $244,58 €$ & $33.340,22 €$ & $10.331,76 €$ & $574,53 €$ \\
\hline FAIRER PREIS ${ }^{\mathrm{a}} /$ Patient:in & $904,94 €$ & $273.389,83 €$ & $66.123,25 €$ & $3.676,97 €$ \\
\hline $\begin{array}{l}\text { FAIRER PREIS }{ }^{\mathrm{a}} \text { pro Jahr } \\
\text { (oder Behandlung, wenn kürzer) }\end{array}$ & $904,94 €$ & 27.338,98€ & $6.612,32 €$ & $3.676,97 €$ \\
\hline Preis EU pro Jahr (Listenpreis) & $35-45.000 €$ & $200-600.000 €$ & $8-60.000 €$ & $25-45.000 €$ \\
\hline $\begin{array}{l}\text { Preis Deutschland pro Jahr } \\
\text { (Listenpreis) }\end{array}$ & $43.000 €$ & $277-601.000 €$ & $8-23.000 €$ & $45.000 €$ \\
\hline
\end{tabular}


Das Modell errechnet einen fairen Preis pro Behandlung von unter $1.000 €$. Zum Vergleich: Der Preis dieser Arzneimittel betrug beim Markteintritt mindestens $25.000 €$ pro Behandlung (Barber et al. 2020). Der verzögerte Zugang zu den entsprechenden Therapien, den viele Patient:innen erdulden mussten, hätte verhindert werden können, wenn man von Anfang an einen fairen Preis angesetzt hätte.

Dieses Beispiel illustriert, wie überaus notwendig es ist, eine Methodik für $\mathrm{F} \& \mathrm{E} \mathrm{zu}$ entwickeln. Die Kalkulation basiert dabei auf den realen F \& E-Aufwendungen, nicht auf der Übernahme anderer Entitäten, und auf den Produktionskosten. Die dokumentierten Produktionskosten dieser chemischen Entitäten liegen dabei übrigens deutlich unter der Pauschale.

\subsubsection{Seltene Erkrankungen am Beispiel von SMA}

Für Orphan Drugs werden oft hohe Preise aufgerufen, die dann auch gezahlt werden. Das hängt damit zusammen, dass man zunächst eine geringe Profitabilität vermutet. Solch hohe Preise blockieren oder verzögern den Zugang zu den Arzneimitteln. Der Blick auf die Spinale Muskelatrophie (SMA) zeigt, dass bei durchaus angemessenen F\&E-Kosten, was bei seltenen Erkrankungen häufiger der Fall ist (Jayasundara et al. 2019), der Preis für Orphan Drugs von zwischen 200.000 und $500.000 €$ pro Patient:in und Jahr bei lebenslangem Einsatz möglicherweise überhaupt nicht gerechtfertigt ist. Das gilt auch dann, wenn die Zahl der Patient:innen sehr begrenzt ist.

Besonders beachtet werden sollte das Aufkommen von Gen- oder Zelltherapien, deren vorgeschlagene Preise darauf beruhen, dass sie Orphan Drugs mit sehr hohen Preisen ersetzen sollen. Unter Berücksichtigung des Refe- renzwerts für die Kosteneffizienz für SMAMedikamente ging der Beirat des niederländischen Instituts für Gesundheitswesen (ZIN) davon aus, dass Nusinersen $85 \%$ günstiger sein sollte als der vom Hersteller veranschlagte Preis (ZIN 2018). Im aktuellen Value-based Pricing erlaubt dieser ungerechtfertigte Listenpreis, für neue Behandlungsformen extrem hohe Preise zu verlangen, die gleichzeitig ,kosteneffektiv" erscheinen. Würde man den fairen Preis des AIM-Algorithmus in Höhe von unter $30.000 €$ pro Behandlungsjahr ansetzen, so würde das den Blick darauf, welche Preise für neue Therapien akzeptabel sind, drastisch ändern.

\subsubsection{Indikationsausweitung am Beispiel von NDM}

Die wissenschaftliche niederländische Plattform „Medicine for Society“ wandte das AIMModell auf Mexiletin an, das für eine neue Indikation zugelassen werden sollte (van den Berg et al. 2021). Dieses Arzneimittel wurde seit langem off-label in der Neurologie für eine seltene neuromuskuläre Erkrankung, die nicht dystophische Myotonie (NDM), eingesetzt. Der Preis lag bei weniger als $249 €$ pro Jahr und Patient:in. Als die Europäische Arzneimittelagentur dem Mittel unter einem neuen Markennamen den Orphan-Status zusprach (EMA 2018), stieg der Preis auf zwischen 30.707 und $60.730 €$ pro Patient:in und Jahr. Medicines for Society errechnete unter Anwendung des AIM-Modells für verschiedene Szenarien einen fairen Preis zwischen 452 und 6.685€. Wie - Tab. 11.1 darstellt, ergibt die Anwendung des AIMAlgorithmus mit den derzeitigen Parametern für diese Indikation einen fairen Preis von $6.612,32 €$. Dies ist ein sehr interessanter Spezialfall. 


\subsubsection{Kostentreiber Krebs}

Die finanzielle Belastung in Zusammenhang mit Onkologika und die Krankheitslast durch Krebs steigen in allen europäischen Gesundheitssystemen enorm. Daher sind die potenziellen Einsparungen, die sich hier durch faire Preise erzielen lassen, einen genaueren Blick wert.

Prof. Uyl De Groot von der Universität Rotterdam wandte ein ähnliches Modell wie das der AIM (Uyl-de Groot und Löwenberg 2018) auf zwei Krebsmedikamente an und zeigte, dass die Onkologie ein weiteres Beispiel für ungerechtfertigte und unfaire Preise ist. Im AIM-Modell liegt der berechnete faire Preis für ein Prostatakrebs-Medikament bei $3.677 €$ pro Patient und Behandlung, was recht nah an dem Preis von 3.094 US-Dollar liegt, der von den Rotterdamer Wissenschaftlern ermittelt wurde. Die aktuellen Marktpreise für das Arzneimittel betragen jedoch mehr als $25.000 €$.

\subsection{Praktische Umsetzung}

\subsubsection{Kurzfristig: Sensibilisierung und Anwendung des Systems auf Iokaler Ebene}

Die AIM hat dieses Modell entwickelt, um einen Beitrag zu aktuellen politischen Debatten über faire Preisfestsetzung und Bezahlbarkeit zu leisten. Außerdem sollen die Öffentlichkeit und die Akteure im Gesundheitswesen für die Möglichkeit alternativer Preise sensibilisiert werden.

Befürworter des Konzepts fairer Preise können das Modell anwenden, um zu ermitteln, wo ein faires Preisniveau wohl liegen könnte. Es ist ein erster Vorschlag, um eine wissenschaftliche, politische und ökonomische Debatte darüber zu initiieren, wie man einen solchen Preis praktisch berechnet. Gleichzeitig soll es helfen, den theoretischen (erheblichen) Spielraum für Preissenkungen bei den Preis- und Kostenerstattungsverhandlungen mit den Herstellern einzuschätzen.

In einem ersten Schritt könnten Entscheidungsträger das Modell praktisch anwenden, um einen neuen „Ankerpreis“ festzulegen und mit diesem fairen Preis im Kopf in die Verhandlungen einzutreten.

Ein sehr nutzerfreundlicher "Simulator" findet sich auf der AIM-Website ${ }^{8}$ (siehe - Abb. 11.3 und 11.4). Er ist frei zugänglich und ermöglicht, den fairen Preis für jedwede neue Arznei oder Indikation zu berechnen. Es müssen nur einige Daten eingegeben oder die vorgeschlagenen Pauschalen verwendet werden. Der Algorithmus errechnet aufgrund dieser Angaben den fairen Preis.

8 https://fairpricingcalculator.eu/. 


\section{EUROPEAN FAIR PRICE Calculator For Medicines}

Welcome to the International Association of Mutual Beneit Societies (AIN)'s fair price calculator for medicines. The calculstor is the practical transcription of AMM's fair pricing model (Ctrl + clik her), using the assumptions for the various parameters made in the model. The caloulator is a tool designed to help heahthcare stakeholders o anybody interested in the matter calculate a FMV price for new or existing medicines (without generic competition) and compare it to the price paid or being negotisted. More importantly this is a hands-on tool with clear proposals for data components to contribute to European and intemational debates about fair pricing and the trarsparency of RQD costs of medicines.

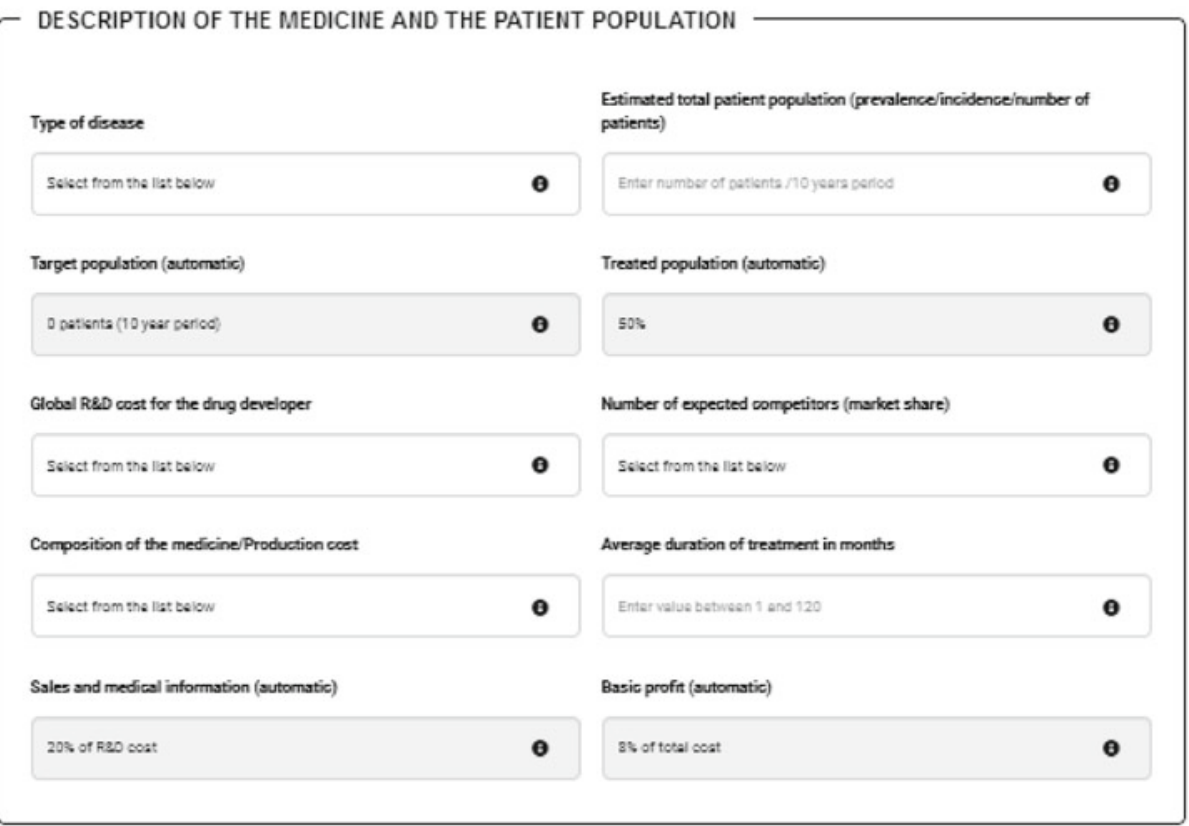

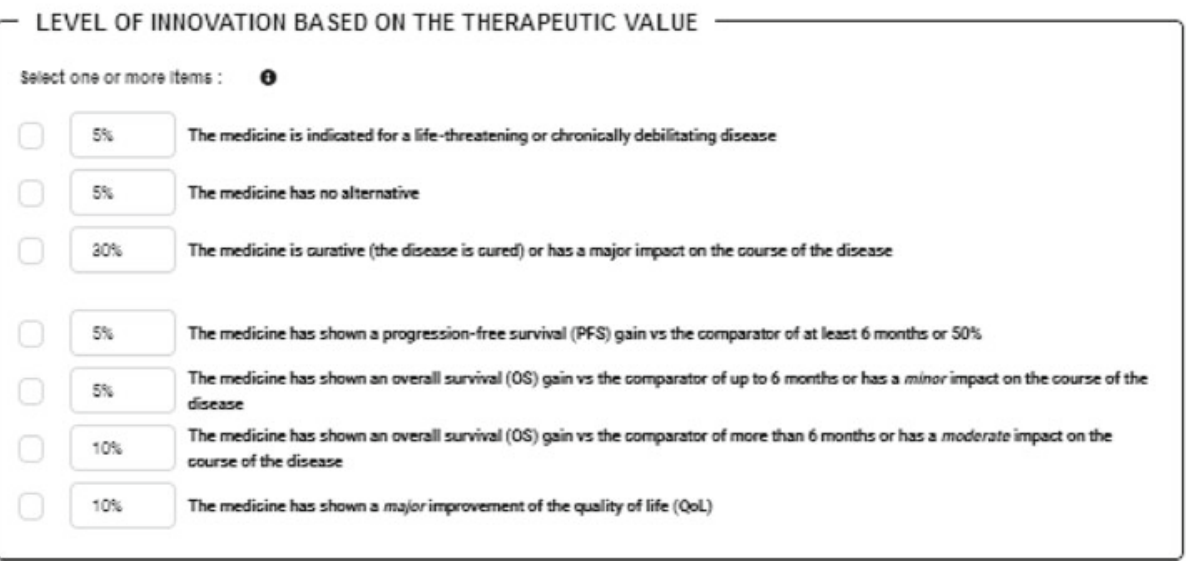

- Abb. 11.3 Homepage des AIM-Simulators für faire Arzneimittelpreise ( $>$ https://fairpricingcalculator.eu/) 


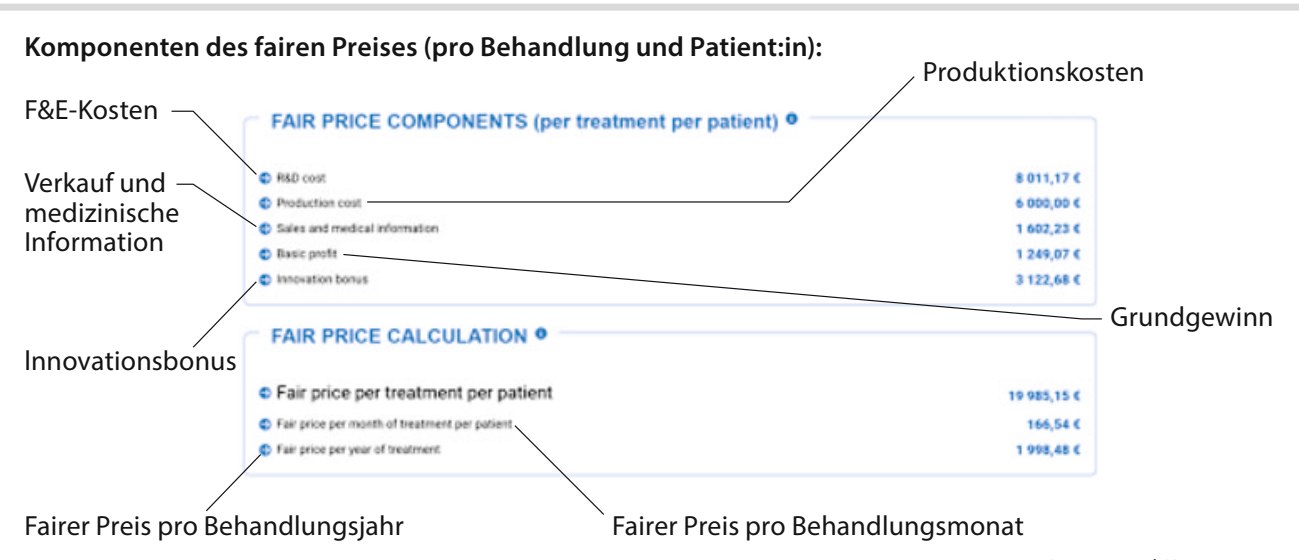

- Abb. 11.4 Details zum errechneten fairen Preis im Kalkulator auf der AIM-Website ( $\downarrow$ https://fairpricingcalculator. $\mathrm{eu} /$ )

\subsubsection{Langfristig: Verknüpfung des fairen Preises mit der Marktzulassung auf EU-Ebene}

Die Organisation der Gesundheitssysteme und die Preisfestsetzung liegen derzeit allein in der Verantwortung der Mitgliedsstaaten. Die Europäische Kommission kann den Preis für die Arzneimittel nicht selbst festlegen. Allerdings kann die Europäische Union die Mitgliedsstaaten dabei unterstützen, die öffentliche Gesundheit zu verbessern. Ein Beispiel für eine solche Unterstützung ist die Rolle der EU bei der Unterzeichnung von Vorverkaufsvereinbarungen mit Herstellern von Covid-19-Impfstoffen, wobei es aber Aufgabe der Mitgliedsstaaten ist, die Impfstoffe zu bestellen.

Die Kalkulation eines fairen Preises und in einem zweiten Schritt die Verpflichtung der Hersteller, Arzneimittel zum berechneten fairen Preis auf den Markt zu bringen, könnte ein Bestandteil der EU-Zulassung für Arzneiprodukte werden. Es würden dann nur Arzneiprodukte in der EU verfügbar gemacht, die einen fairen Preis haben und diesen nicht überschreiten.
Der faire Preis müsste zum Zeitpunkt der Marktzulassung oder kurz danach festgesetzt werden, um den Markteintritt und -zugang nicht zu verzögern. Der Artikel 6 der Richtlinie 2001/83/EC zum Gemeinschafts-Kodex in Bezug auf Humanarzneimittel könnte somit ergänzt werden um Hinweise zu den Angaben, die die Europäische Kommission für die Kalkulation eines fairen Preises benötigt.

Bestehende europäische Behörden könnten zur Festsetzung eines solchen fairen Preises beitragen. Hersteller, die eine Marktzulassung beantragen, würden neben den erforderlichen Daten für die Registrierung der Indikation sowohl F\&E- als auch Produktions- und Gemeinkosten an die Europäische Arzneimittelbehörde (EMA) übermitteln. Die EMA würde diese Daten analysieren und validieren. Die Komponenten des Innovationsbonus, die sich auf den therapeutischen Nutzen beziehen und mittels Health Technology Assessment (HTA) bewertet werden müssen, wären nicht Teil der EMA-Evaluation, sondern würden von einer (nationalen oder europäischen) Stelle mit HTA-Kompetenz beurteilt.

Die Einführung in Europa könnte Schritt für Schritt erfolgen, beginnend mit Produkten mit Indikationserweiterung und überhöh- 
ten Preisen und Arzneimitteln, die voraussichtlich das Budget stark belasten werden.

Es gibt jedoch ein paar Unwägbarkeiten hinsichtlich der Machbarkeit eines solchen Preisfestsetzungsrahmens auf EU-Ebene. Preisfestsetzung und Erstattung fallen laut EUVerträgen in die Zuständigkeit der Mitgliedsstaaten. Es könnte daher schwierig werden, das EU-Recht entsprechend zu ändern. Die Debatten im Zusammenhang mit der Verabschiedung der HTA-Richtlinie legen nahe, dass es heikel ist, eine Einigung über gemeinsame Maßnahmen mit direkten Auswirkungen auf die Preisfindung und Erstattung auf nationaler Ebene zu erzielen. Allerdings konnte die EU im Rahmen ihrer Unterstützungsfunktion bei der Organisation der nationalen Gesundheitssysteme bereits zu einigen Elementen des Modells einen Beitrag leisten, etwa bei der Kostendokumentation und der Methodik zur Berechnung dieser Kosten. Solange ein in Europa voll harmonisiertes HTA fehlt, lassen sich zumindest auf regionaler Ebene Fortschritte bei den Bewertungen erzielen. In den vergangenen Jahren haben sich einige Mitgliedsstaaten an einer Reihe von freiwilligen Kooperationen bei der gemeinsamen Bewertung der Preisfestsetzung von Arzneimitteln beteiligt (Eatwell und Swierczyna 2019). Die Mitgliedsstaaten könnten auch ermutigt werden, den fairen Preis als alternative Grundlage bei den Preisfestsetzungs- und Vergütungsverhandlungen einzusetzen. Die Europäische Kommission könnte - etwa über neue oder bereits existierende Plattformen wie EURIPID - faire Benchmark-Preise für verschiedene Krankheitsarten entwickeln und in Umlauf bringen.

\subsection{Der Weg nach vorn: Transparenz und Methodik}

Um die kurz- und langfristigen Ziele zur Einführung fairer Preise in Europa zu erreichen, sind unterstützende Maßnahmen notwendig.
Anstrengungen, die die Kosten- und Preistransparenz verbessern, sollten gefördert werden. Ein Beispiel dafür ist die WHO-Resolution „Verbesserung der Transparenz der Märkte für Arzneimittel, Impfstoffe und andere Gesundheitsprodukte“, die am 8. Mai 2019 verabschiedet wurde (WHO 2019). Zur Transparenz des Arzneimittelmarktes gehört es dabei auch, einen leichten Zugang zu Datenbanken zu ermöglichen, die Aufschluss geben über die Zahl der Patient:innen, die jeweils von einer bestimmten Krankheit bzw. von einer spezifischen Indikation betroffen sind.

Gleichzeitig sollten die europäischen Akteure im Rahmen der ,Pharmazeutischen Strategie für Europa" eine Methodik zur Übermittlung von Kosten (F \& E - inklusive Übernahmen - und Produktions- und Gemeinkosten) und Festlegung von akzeptablen Gewinnmargen entwickeln. Wenn man die Auswirkungen von Preissenkungen auf den Zugang zu Arzneimitteln in jedem Mitgliedsstaat evaluierte, dann würden die potenziellen finanziellen und gesellschaftlichen Auswirkungen fairer Preise wissenschaftlich dokumentiert. Es würde wohl auch die Annahme des Modells stützen, dass Preissenkungen das Volumen erhöhen und ein neues, akzeptables Gleichgewicht für die Pharmaindustrie schaffen.

Auf globaler Ebene sollte es eine offene Debatte mit der pharmazeutischen Industrie darüber geben, wie sie gleichzeitig die Nachhaltigkeit der Gesundheitssysteme unterstützen und angemessene Profite erzielen kann und wie man einen Interessensausgleich erreicht. Es ist zwingend notwendig, dem pharmazeutischen Sektor klare Signale zu senden, um eine langfristige Perspektive für deren Investitionen zu gewährleisten. Die mithilfe der derzeitigen hohen Preise erzielten Einnahmen sollten vor allem in F \& E investiert werden und nicht in andere Strategien wie Aktienrückkäufe (Gibney und Woleben 2021). Es müsste eine große politische Debatte in Gang gesetzt werden, um sinnvolle Veränderungen auf diesem Gebiet und somit Bezahlbarkeit und faire Preisgestaltung bei Arzneimitteln zu erreichen. 


\subsection{Fazit}

Vor dem Hintergrund zunehmender Besorgnis in Hinblick auf den Zugang zu einigen neuen Arzneimitteln ist ein Ausweg aus überteuerten Arzneimittelpreisen möglich, wenn die EU27Staaten sich zusammentun und gemeinsam eine Alternative zum bisherigen Preisfestsetzungssystem vorschlagen. Aufgrund der hohen Preise hat Value-based Pricing zu Problemen beim Zugang zur Behandlung geführt und zu einer Konzentration von F\& E auf eine begrenzte Zahl von Krankheiten, während große Bereiche weiterhin einen ungedeckten medizinischen Bedarf aufweisen.

Würde man eine Verbindung zwischen dem Preis, den Kosten für das Pharmaunternehmen und dem therapeutischen Nutzen eines innovativen Arzneimittels herstellen, so würde dies die Kosten für die Gesundheitssysteme senken. Gleichzeitig würde der Zugang für die Patient:innen verbessert und nützliche Neuentwicklungen würden dennoch gefördert.

Die Kostenträger wären bei Preisverhandlungen in einer besseren Position, wenn sie den transparenten AIM-Algorithmus benutzten, denn dieser kombiniert die Kosten des Arzneimittels, einen auskömmlichen Gewinn und einen „Innovationsbonus“, der den therapeutischen Nutzen für die Patient:innen berücksichtigt. Die Gegenüberstellung des fairen Preises als Ausgangspunkt für Verhandlungen mit der Preisvorstellung des Herstellers würde die wichtige Diskussion darüber befördern, was denn überhaupt ein fairer Preis ist.

Mit ihrem Modell hat die AIM ein Instrument vorgeschlagen, mit dem sich auch Gesetze oder regulatorische Maßnahmen anstoßen lassen, die die Bezahlbarkeit von Arzneimitteln überhaupt erst ermöglichen.

\section{Literatur}

AIM - Association Internationale de la Mutualité (2021) AIM bietet ein Instrument zur Berechnung gerechter und transparenter Preise in Europa für zugängliche pharmazeutische Innovationen. https:// www.aim-mutual.org/wp-content/uploads/2021/07/ AIMfairpricingModel_June2021_FINAL_DE.pdf. Zugegriffen: 11. Juli 2021

Akcigit U, Chen W, Díez F, Duval R, Engler P, Fan J, Maggi C, Tavares M, Schwarz D, Shibata I, Villegas-Sánchez C (2021) Rising corporate market power: emerging policy issues. https://www.imf. org/-/media/Files/Publications/SDN/2021/English/ SDNEA2021001.ashx. Zugegriffen: 25. Apr. 2021

Bach P (2019) 'Anchoring' was at work in setting the price of Novartis' new gene therapy. https://www.statnews. com/2019/06/04/anchoring-price-zolgensma/. Zugegriffen: 4. Mai 2021

Barber M, Gotham D, Khwairakpam G, Hill A (2020) Price of a hepatitis $\mathrm{C}$ cure: cost of production and current prices for direct-acting antivirals in 50 countries. J Virus Erad 6(3):100001. https://doi.org/10. 1016/j.jve.2020.06.001 (https://www.sciencedirect. com/science/article/pii/S2055664020300017?via \%3Dihub. Zugegriffen: 4. Mai 2021)

Eatwell E, Swierczyna A (2019) Emerging voluntary cooperation between European healthcare systems: are we facing a new future? Med Access @ Point Care. https://doi.org/10.1177/2399202619852317

EFPIA - European Federation of Pharmaceutical Industries and Associations (2019) The pharmaceutical industry in figures - key data 2019. https://www. efpia.eu/media/412931/the-pharmaceutical-industryin-figures-2019.pdf. Zugegriffen: 4. Mai 2021

European Medicines Agency (2018) Namuscla, European public assessment report. https://www.ema. europa.eu/en/medicines/human/EPAR/namuscla. Zugegriffen: 19. Aug. 2021

Gibney M, Woleben J (2021) Big Pharma rallies on share buybacks as most companies cut back during pandemic. https://www.spglobal.com/marketintelligence/ en/news-insights/latest-news-headlines/big-pharmarallies-on-share-buybacks-as-most-companies-cutback-during-pandemic-62845839. Zugegriffen: 4. Mai 2021

Harvard Law School, Program on Negotiation (2019) The anchoring effect and how it can impact your negotiation. https://www.pon.harvard.edu/daily/negotiationskills-daily/the-drawbacks-of-goals/. Zugegriffen: 27. Apr. 2021

Jayasundara K, Hollis A, Krahn M, Mamdani M, Hoch J, Grootendorst P (2019) Estimating the clinical cost of drug development for orphan versus nonorphan drugs. Orphanet J Rare Dis 14:12. https:// doi.org/10.1186/s13023-018-0990-4 (https://ojrd. biomedcentral.com/articles/10.1186/s13023-0180990-4\#citeas. Zugegriffen: 4. Mai 2021)

Morgan SG, Bathula HS, Moon S (2020) Pricing of pharmaceuticals is becoming a major challenge for health systems. BMJ 368:14627. https://doi.org/10. 
1136/bmj.14627 (https://www.bmj.com/content/368/ bmj.14627. Zugegriffen: 27. April 2021)

Prasad V, Mailankody S (2017) Research and development spending to bring a single cancer drug to market and revenues after approval. J Am Med Assoc Intern Med 177(11):1569-1575. https://doi.org/ 10.1001/jamainternmed.2017.3601

Popa C, Holvoet K, Van Montfort T, Groeneveld F, Simoens S (2018) Risk-return analysis of the biopharmaceutical industry as compared to other industries. Front Pharmacol 9:1108. https://doi.org/10. 3389/fphar.2018.01108/full

Ran T, Eichmüller S, Schmidt P, Schlander M (2020) Cost of decentralized CAR T-cell production in an academic nonprofit setting. Int J Cancer 147:3438-3445. https://doi.org/10.1002/ijc.33156

Uyl-de Groot C, Löwenberg B (2018) Sustainability and affordability of cancer drugs: a novel pricing model. Nat Rev Clin Oncol. https://doi.org/10.1038/s41571018-0027-X

van den Berg $\mathrm{S}$, van der Wel V, de Visser S, Stunnenberg B, Timmers L, van der Ree M, Postema P, Hollak C (2021) Cost-based price calculation of Mexiletine for Nondystrophic Myotonia. Value Health. https://doi.org/10.1016/j.jval. 2021.02.004 (https://www.sciencedirect.com/science/ article/pii/S1098301521001431. Zugegriffen: 4. Mai 2021)

van der Gronde T, Uyl-de Groot C, Pieters T (2017) Addressing the challenge of high-priced prescrip- tion drugs in the era of precision medicine: A systematic review of drug life cycles, therapeutic drug markets and regulatory frameworks. PloS one 12(8):e0182613. https://doi.org/10.1371/journal. pone. 0182613

Wenzl M, Chapman S (2019) Performance-based managed entry agreements for new medicines in OECD countries and EU member states: How they work and possible improvements going forward. OECD Health Working Papers No 115. OECD Publishing, Paris https://doi.org/10.1787/6e5e4c0f-en

WHO - World Health Organization (2017) Fair pricing forum Q\&A. https://www.who.int/medicines/access/ fair_pricing/q_a-fair_pricing_meds/en/. Zugegriffen: 25. Apr. 2021

WHO - World Health Organization (2019) Improving the transparency of markets for medicines, vaccines, and other health products. https://apps.who.int/gb/ebwha/ pdf_files/WHA72/A72_ACONF2Rev1-en.pdf. Zugegriffen: 5. Mai 2021

ZIN - Zorginstituut Nederland (2018) Pakketadvies nusinersen (Spinraza®). https://www. zorginstituutnederland.nl/binaries/zinl/documenten/ adviezen/2018/02/07/pakketadvies-nusinersenspinraza-voor-de-behandeling-van-spinalemusculaire-atrofie-sma/Brief+pakketadvies+ nusinersen+bij+SMA+aan+minister+van+medische+ zorg+en+sport.pdf. Zugegriffen: 5. Mai 2021

Open Access Dieses Kapitel wird unter der Creative Commons Namensnennung 4.0 International Lizenz (http:// creativecommons.org/licenses/by/4.0/deed.de) veröffentlicht, welche die Nutzung, Vervielfältigung, Bearbeitung, Verbreitung und Wiedergabe in jeglichem Medium und Format erlaubt, sofern Sie den/die ursprünglichen Autor(en) und die Quelle ordnungsgemäß nennen, einen Link zur Creative Commons Lizenz beifügen und angeben, ob Änderungen vorgenommen wurden.

Die in diesem Kapitel enthaltenen Bilder und sonstiges Drittmaterial unterliegen ebenfalls der genannten Creative Commons Lizenz, sofern sich aus der Abbildungslegende nichts anderes ergibt. Sofern das betreffende Material nicht unter der genannten Creative Commons Lizenz steht und die betreffende Handlung nicht nach gesetzlichen Vorschriften erlaubt ist, ist für die oben aufgeführten Weiterverwendungen des Materials die Einwilligung des jeweiligen Rechteinhabers einzuholen.

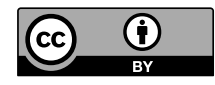

